\section{Intersensory effects in the psychological refractory period*}

\author{
IRA H. BERNSTEIN and MARK H. CLARK \\ University of Texas at Arlington, Arlington, Texas 76010
}

Two experiments examined reaction time (RT) to each of two stimulus events separated by short interstimulus intervals (ISI). The essential contrast was RT to the second visual signal, $\mathrm{RT}_{2}$, in auditory-visual (A-V) vs visual-visual (V-V) sequences. With response, certain pairings in Experiment 1, an effect apparently demonstrating a single-channel process (Welford, 1952), was noted. $\mathrm{RT}_{2}$ was generally faster for $\mathrm{A} \cdot \mathrm{V}$ as opposed to V.V sequences especially when Ss were uncertain as to the sequence that would occur. At 0 -msec ISI, the $\mathrm{RT}_{2}$ difference between sequences approached the $\mathrm{RT}_{1}$ difference. More rapid $\mathrm{RT}_{2}$ to $\mathrm{A} \cdot \mathrm{V}$ sequences was also observed with go vs no-go pairings in Experiment 2 when the initial event was a go signal. However, the RT difference disappeared upon error correction, making the $\mathrm{RT}_{2}$ sequence difference of questionable relevance to the hypothetical single-channel process. $\mathrm{RT}_{2}$ was more rapid following a null no-go signal when the no-go signal was contrasted with a visual as opposed to auditory go signal. The latter effect was independent of error and is consistent with channel-switching theory (Kristofferson, 1967).

Consider the following reaction-time (RT) experiment. Two successive stimulus events $\left(S_{1}\right.$ and $\left.S_{2}\right)$ are presented with a short interstimulus interval (ISI) separating their onsets. In one case, an auditory event is followed by a visual event (A-V sequence). In a second case a visual event is followed by another visual event (V-V sequence). Assume that the initial RT $\left(R T_{1}\right)$ is more rapid when $S_{1}$ is auditory as opposed to visual. What might we predict about RT to the subsequent visual event, $\mathrm{RT}_{2}$, when short ISIs (less than a normal RT) are employed?

On the basis of single-channel information-processing theory (Welford, 1952), the processing of the second signal cannot begin until the response to the first signal is made. A strong prediction is therefore that $\mathrm{RT}_{2}$ in the $\mathrm{A} \cdot \mathrm{V}$ sequence will be faster than $R T_{2}$ in the V.V sequence by the same amount as the $\mathrm{RT}_{1}$ difference. This effect would arise simply because the reduction in "throughput" time for an auditory $S_{1}$ could be passed onto $S_{2}$ directly; apart from this effect there would be no systematic differences in $\mathrm{RT}$ or error rate peculiar to intersensory vs intrasensory presentation.

On the other hand, assume that it takes time to switch from one modality to

*Supported by NIMH Grant 12530-02 to the senior author and by the University of Texas at Arlington Liberal Arts Organized Research Budget. The authors are grateful to: Dr. John Heslip and Dr. R. Gottsdanker for their comments, and to the Physics Department at the University of Texas at Arlington, Dr. Aram Glorig. Dallas Hearing and Speech Center, and Mr. David Broyles for their help in calibrating the equipment. A version of the present paper was presented at the 1968 meetings of the Psychonomic Society. another. For example, Kristofferson (1967) has proposed that channel switching can occur only at certain discrete instants, approximately each $50 \mathrm{msec}$, with a time base asynchronous to stimulus input. On this basis there would be a penalty upon $\mathrm{RT}_{2}$ in the $\mathrm{A} \cdot \mathrm{V}$ case. Depending upon additional assumptions, the penalty would be either 25 or $50 \mathrm{msec}$ at 0 -msec ISI and would decline with ISI in either case.

The first experiment sought to discriminate between these two alternative approaches to intersensory effects upon simple RT. The second experiment improved upon the method of the first experiment.

\section{EXPERIMENT 1}

Method

Variables. In addition to sequence (A-V vs V.V) and ISI $(0,25,50,75$, and $100 \mathrm{msec}$ ), we also manipulated the channel certainty. Specifically, in channel-certain (CC) conditions, Ss had foreknowledge of the sequence type within a given block of trials; in the channel-uncertain conditions (CU), the two sequence types were randomized and Ss had no foreknowledge.

Subjects. Two advanced undergraduate students (RF) and (HH), who had prior experience in PRP experiments, and one graduate student (BE), who had not previously served in an RT experiment, comprised the sample.

Procedure. Following practice, $200 \mathrm{RT}$ pairs were obtained for each level of channel certainty, ISI, and sequence type. For V.V sequences in the $\mathrm{CC}$ condition, a block consisted of 50 trials at a given ISI with either a left-right order or the reverse. Order was constant within a block but counterbalanced across blocks. For A.V sequences in the $\mathrm{CC}$ condition, a binaural tone replaced the first visual event. In the CU condition, trials could consist of either

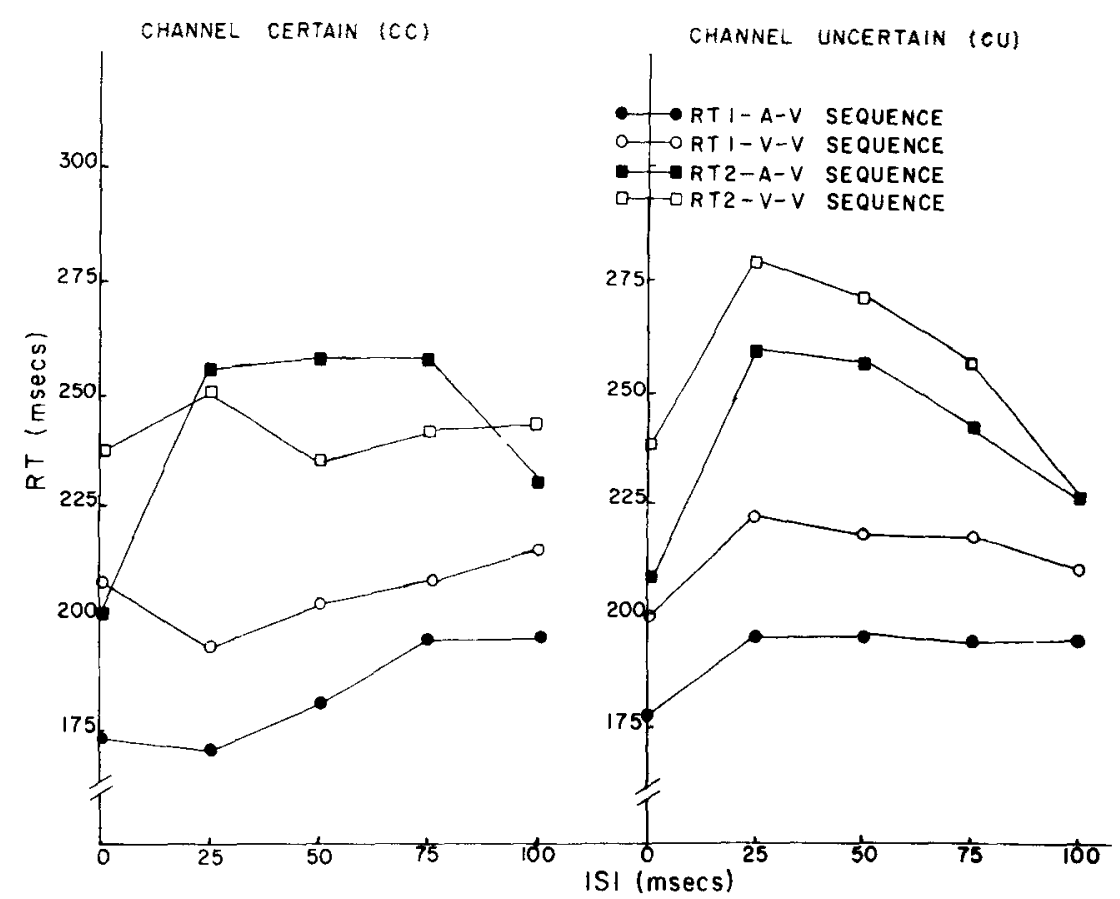

Fig. 1. $R T_{1}$ and $R T_{2}$ as a function of sequence and ISI, separately for the channel certain and channel uncertain conditions, Experiment 1 . 
random probability. Ss made the same responses regardless of the sequence type. As order was again fixed within a block, response uncertainty was never present. The instructions paralleled those used by Bernstein, Blake, and Hughes (1968) and included telling Ss to attempt to maintain a constant $\mathrm{RT}_{1}$ across ISIs. The ISI within a given block was demonstrated and labeled. In the CU conditions, both sequences were shown to the Ss. One event was arbitrarily designated $S_{1}$ at 0 msec.

Apparatus and stimuli. The apparatus and stimuli were as described by Bernstein et al (1968), with the addition of an RCA audio signal generator and a pair of Koss PRO 4 headphones. The visual events were the onset of a $45-\mathrm{min}$ spot of light produced by the back illumination of a hole cut in an opaque card located in the front receptacles of a Scientific Prototype Model GB tachistoscope. The blank field held the fixation spot $(18 \mathrm{fc})$, which was normally on except for a .1-sec offset, serving as the warning signal for the $2-\mathrm{sec}$ foreperiod delay. The remaining fields held the two visual events (12 fc each), which were symmetrically placed on the imaginary horizontal axis of the fixation spot. With all stimulus events on, the display width subtended $3 \mathrm{deg} 20 \mathrm{~min}$ of arc. The auditory event that replaced the first visual event on $A \cdot V$ trials was a $90-d B$ $1-\mathrm{kHz}$ sinusoidal signal delivered binaurally and in phase. All discriminative stimuli were $20-\mathrm{msec}$ pulses of light or sound.

\section{Results}

The experimental findings are summarized in Fig. 1. Across both conditions of channel certainty (CC and $\mathrm{CU})$ there is a consistent difference in favor of faster $\mathrm{RT}_{1}$ to initial auditory events. The average difference was $25 \mathrm{msec}$ and significant $(\mathrm{p}<.001)$ both within $\mathrm{Ss}$ (individual $t$ tests) and between $S s$ (Conditions by Sequence by Ss analysis of variance). $\mathrm{CC}$ led to slightly more rapid responding to $S_{1}$ than did $\mathrm{CU}$ by $8 \mathrm{msec}$ $(p<.05)$. There was also an interaction of channel certainty with ISI upon $\mathrm{RT}_{1}$ $(\mathrm{p}<.05)$. With $\mathrm{CC}, \mathrm{RT}_{1}$ varied erratically across ISI. With $\mathrm{CU}, \mathrm{RT}_{1}$ showed an initial rise between 0 and $25 \mathrm{msec}$ ISI but remained level thereafter. Across conditions of channel certainty $\mathrm{RT}_{2}$ is consistently longer than $\mathrm{RT}_{1}$, demonstrating a refractory effect. Exceptions for V.V occurred for $\mathrm{CC}$ at 0 -msec ISI which may be due to a strategy of joint responding to the combined $S_{1}+S_{2}$ event at $0 \mathrm{msec}$ vs sequential responding to the separate events at the other ISIs. Other evidence supporting this difference in strategy is seen in the relatively large rise in $\mathrm{RT}_{2}$ from 0 to $25 \mathrm{msec}$ for $\mathrm{A} \cdot \mathrm{V}$ sequences.
Most relevant to our initial hypothesis is the comparison between $\mathrm{RT}_{2}$ for the $\mathrm{A}-\mathrm{V}$ and $\mathrm{V} \cdot \mathrm{V}$ sequences (closed vs open squares in Fig. 1). A "pure" single-channel prediction would yield a difference in favor of (i.e., more rapid RT) the filled squares by an amount equal to the $\mathrm{RT}_{1}$ difference between auditory and visual $S_{1}$ events. A pure channel-switching prediction (without consideration of single-channel effects) would yield a difference in favor of the open squares. Neither prediction holds consistently for the $\mathrm{CC}$ condition. At $0 \mathrm{msec}$ the single-channel prediction appears to hold, while at 50 -msec ISI the switching prediction appears to hold. In the $\mathrm{CU}$ condition, however, the data lean heavily toward the single-channel prediction, particularly at the shorter ISIs. The $\mathrm{RT}_{2}$ differences (A-V vs V-V) are 32 , $27,20,13,14$, and $4 \mathrm{msec}$, as contrasted with $\mathrm{RT}_{1}$ differences of $32,20,13,14$, and $16 \mathrm{msec}$. The overall difference between these differences is not statistically significant, although significance was present in some individual cases at 100 -msec ISI, where single-channel effects might begin to disappear.

The data generally replicate a prior study concerned with sequential simple visual RT, which employed the same ISIs (Bernstein et al, 1968, Experiment 2). The difference between the studies is that response-terminated signals were employed in the former and pulsed signals were employed in the latter. Both studies obtained a refractory effect and a rise in $\mathrm{RT}_{1}$ with ISI, indicating that refractory effects can be obtained with minimal time and event uncertainty.

The data thus apparently demonstrate a single-channel effect while providing minimal support for a switching position. However, there was a fair amount of data "noise," especially in the CC condition, so the results can at best be considered preliminary. In particular, part of the noise seems to have arisen from different response strategies adopted by different $\mathrm{Ss}$, ranging from strict serial processing to joint responding. In some cases $\mathrm{Ss}$ reported in postexperimental interview that they changed strategies with ISI. Also, the design was inadequate to insure that Ss had attended to both events. The apparent single-channel effect at shorter ISIs can be explained by assuming that Ss based their second responses upon $S_{1}+$ the estimated ISI, rather than upon the nominal stimulus event, $S_{2}$. This strategy would lead to more rapid $R T_{2}$ in $A \cdot V$ sequences for the same reasons that more rapid $R T_{1}$ would be obtained. Errors in time estimation would produce discrepancies at $100-\mathrm{msec}$ ISI. No switching effects would be present because Ss would remain aligned to the modality on which $\mathrm{S}_{1}$ occurred.

\section{EXPERIMENT 2}

Stimulus uncertainty in the form of a go vs no-go discriminateon was introduced in an attempt to insure that both stimulus events were attended to. The particular task was an extention of one previously employed by Bernstein, Blake, and Clark (1970). A given trial could consist of four possible events as $S_{1}$ could occur $\left(S_{1}+\right)$ or not occur $\left(S_{1}-\right)$ independently of the occurrence $\left(S_{2}+\right)$ or nonoccurrence $\left(S_{2}-\right)$ of $S_{2}$. Bernstein et al (1970), using visual S+ and null S- events, obtained two classes of effects. In the first class, changes in RT to $S+$ occurred which were independent of changes in the probability of inhibiting to $\mathrm{S}-, \mathrm{P}(\mathrm{I})$. In the second class, changes in RT occurred which were accompanied by corollary changes in error rate. Differences between $\mathrm{RT}_{1}$ and $\mathrm{RT}_{2}$ fell in the first category, which will be termed a "sensitivity" effect through analogy with the signal-detection literature, denoting a change in hit rate that is not dependent upon a change in false-alarm rate. The probability that a trial would be a go trial, $\mathrm{P}(\mathrm{S})$, provided an example of a "decisional" effect, again a term used by analogy to effects observed in signal detection where hit rate and false alarm rate changes occur concomitantly. Bernstein et al (1970) made the distinction analytically through linear-regression techniques. A scatter plot of RT vs P(I) was obtained for each data point. The obtained plot was clearly nonlinear as RT changed less rapidly for values of $P(I)$ between .7 and .8 as opposed to .9 and 1.0 , even though higher values of RT were generally associated with higher values of $\mathrm{P}(\mathrm{I})$. Transformation of $\mathrm{P}(\mathrm{I})$ into normal deviate $(Z)$ resulted in a reasonably linear scatter plot allowing RT to be adjusted for $Z$. RT effects that are present after adjustment for $Z$ are thus sensitivity effects, whereas those that disappear following correction are decisional effects.

Experiment 2 can thus be summarized in terms of three objectives: (1) To see if a switching effect would be obtained by forcing $S$ s to attend to both stimulus events; (2) to attempt to replicate the single-channel effect obtained in Experiment 1; and (3) given that Objectives 1 and/or 2 were met, to see if they were sensitivity or decisional in nature.

\section{Method}

Variables. The basic variables were sequence (A-V vs V-V), ISI $(0,20,40$, and $60 \mathrm{msec}$ ), and $P(S)$. The ISIs were chosen to explore more fully the range where sequence effects occurred in Experiment 1 . $\mathrm{S}_{1}+$ and $\mathrm{S}_{2}+$ occurred independently with $\mathrm{P}(\mathrm{S})=.4$ in one condition and .8 at another. Bernstein et al (1970) found that 


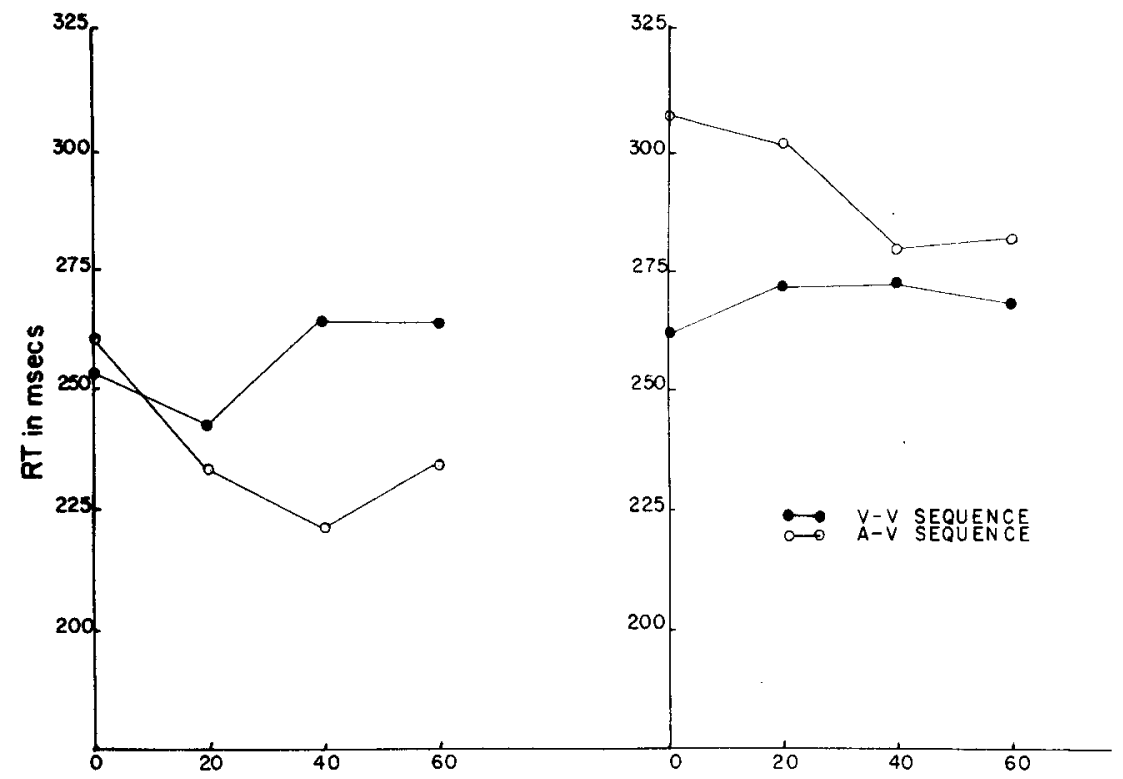

ISI in msecs

Fig. 2. $R T_{2}$ as a function of sequence and ISI, separately for $S_{1}+$ and $S_{1}$-, Experiment 2 .

there was an inverse relation between $P(S)$ and both RT and $\mathrm{Z}$. Thus allowing $\mathrm{P}(\mathrm{S})$ to vary increases the magnitude of the correlation between $R T$ and $Z$ and facilitates the task of obtaining regression lines for error correction purposes. Finally, trials were divided into those on which the alternative event was a go vs a no-go event, i.e., $\mathrm{RT}_{2}$ was obtained separately for $\mathrm{S}_{1}+$ and $S_{1}-$ and vice versa for $\mathrm{RT}_{1}$.

Subjects. The Ss were three advanced undergraduate males majoring in psychology at the University of Texas at Arlington. One (HH) had served in Experiment 1, the others (CN and DM) had served in prior PRP experiments.

Procedure. A total of 384 trials were run in two blocks (one per session) at each level of $P(S)$, sequence, and ISI for each $S$. Presentation was thus channel certain in the sense that $S$ knew the $S_{1}+$ event that would occur (auditory or visual) if an $\mathrm{S}_{1}+$ event did, in fact, occur. The instructions were changed so that $S$ was shown the sequence on each block of trials, but the ISI was not labeled. He was still instructed to attempt to maintain independence of choice but not necessarily to maintain equal $\mathrm{RT}_{1}$ across ISIs, as $\mathrm{RT}_{1}$ equality across ISIs need not actually characterize S's performance capabilities. Because error rates could be obtained and corrected for, it was felt unnecessary to introduce such artificial instructional constraints.

Apparatus and stimuli. The apparatus and stimuli were the same as used in Experiment 1 except for two modifications: (1) The control circuitry was modified to allow $\mathrm{E}$ to inhibit either or both stimulus events, and (2) the auditory signal was delivered monaurally to the homolateral ear on which responding would occur.

\section{Results}

Inspection of the data indicated that the trends observed for the .4 and .8 conditions were equivalent in that both $\mathrm{P}(\mathrm{I})$ and RT were higher in the former case but trends for experimental manipulations were common to both conditions. As a result, data from the two conditions were pooled for purposes of data presentation. Preliminary analyses indicated that RT varied more linearly with $Z$ than with $P(I)$, as it did previously (Bernstein et al, 1970). Consequently, inhibition data will be discussed in terms of the transformed measures, $Z_{1}$ and $Z_{2}$. The inferential tests to be reported were analyses of variance of RT means and $Z$, separately for $S_{1}$ and $S_{2}$ (sequence, presence vs absence of alternative event, ISI, and Ss).

Analysis of variance of condition means indicated that $\mathrm{RT}_{1}$ was more rapid for the A.V as opposed to V-V sequences, as before $(\mathrm{p}<.001)$. Also, $\mathrm{RT}_{1}$ was more rapid when the succeeding event was a go as opposed to a no-go event. Thus, Ss responded more rapidly to the first event when they also had to react overtly rather than inhibit to the second. Response interdependence was also found for $\mathrm{RT}_{2} . \mathrm{S}$ reacted more rapidly to $S_{2}+$ following $S_{1}+$ as opposed to $\mathrm{S}_{1}-(\mathrm{p}<.01)$. A trend towards higher $Z_{1}$ when $S_{2}$ - occurred was not significant but the converse was as $Z_{2}$ was higher for $S_{1}$ - as opposed to $S_{1}+$ $(p<.01)$. Thus, corresponding decisions to react vs not to react were generally easier to make than opposing decisions to the two events. Finally, $\mathrm{RT}_{1}$ declined with ISI $(\mathrm{p}<.01)$.

The data regarding sequence effects are contained in Fig. 2, which presents $\mathrm{RT}_{2}$ as a function of A-V (open points) vs V-V (filled points) sequences, separately for those trials on which the first event did $\left(S_{1}+\right)$ vs $\left(S_{1}-\right)$ did not occur. To be noted is that the physical stimulation was identical on $S_{1}$ trials (right side of graph) in $A \cdot V$ and $V \cdot V$ sequences; only the expectations as to what affirmative first events could have occurred but did not separated the two sets of data points. With $\mathrm{S}_{1}+$ (left side of graph) effects similar to those obtained in Experiment 1 were found in that $\mathrm{RT}_{2}$ was more rapid for $\mathrm{A}-\mathrm{V}$ (open points) as opposed to V-V (filled points) sequences. However, the converse was found for those trials on which $S_{1}$ occurred. Ss could react more rapidly to a visual event following the nonoccurrence of a possible visual event than a possible auditory event. $\mathrm{RT}_{2}$ also declined more rapidly for $A \cdot V$ as opposed to $V \cdot V$ sequences. The $S_{1}+$ vs $S_{1}-$ by ISI, the $S_{1}-$ vs $S_{1}+$ by Sequence, and the Sequence by ISI interactions were all significant beyond the .01 level.

The inhibition data for $S_{2}-$ paralleling the RT data for $S_{2}+$ are presented in Fig. 3. The more rapid RT found for A.V sequences on $S_{1}+$ trials was accompanied by a lower probability of inhibition. On the other hand, there is no systematic sequence difference in $\mathrm{Z}_{2}$ on $\mathrm{S}_{1}$ - trials. This finding for $S_{1}-$ indicates that $S$ s have a diminished ability to react to a signal occurring on a modality different from a prior signal. This RT difference to $S_{2}+$ is not offset by a lower error rate to $S_{2}-$.

Regression lines were obtained for the RT data, based upon 32 paired data points [the two levels of $P(S)$ by $S_{1}+$ vs $S_{1}-$ by four ISIs by two Sequence Types]. The correlations between $\mathrm{RT}_{2}$ and $\mathrm{Z}_{2}$ ranged from .50 to .63 across $S_{2}$ (all $\left.p<.005\right)$, allowing the linear regression technique used by Bernstein et al (1970) to remove the effects of $Z$ from $R T$.

The corrected RT means are presented in Fig. 4, where it may be seen that there is no sequence effect on $S_{1}+$ trials; the slight trend towards more rapid responding on V.V trials was nonsignificant. Conversely, the difference on $\mathrm{S}_{1}$ - trials favoring $\mathrm{RT}$ to $\mathrm{V}-\mathrm{V}$ sequences remained intact and is of a magnitude consistent with Kristofferson's specific theory of channel switching. The main effect of sequence type and the interaction between sequence type and $S_{1}+$ 


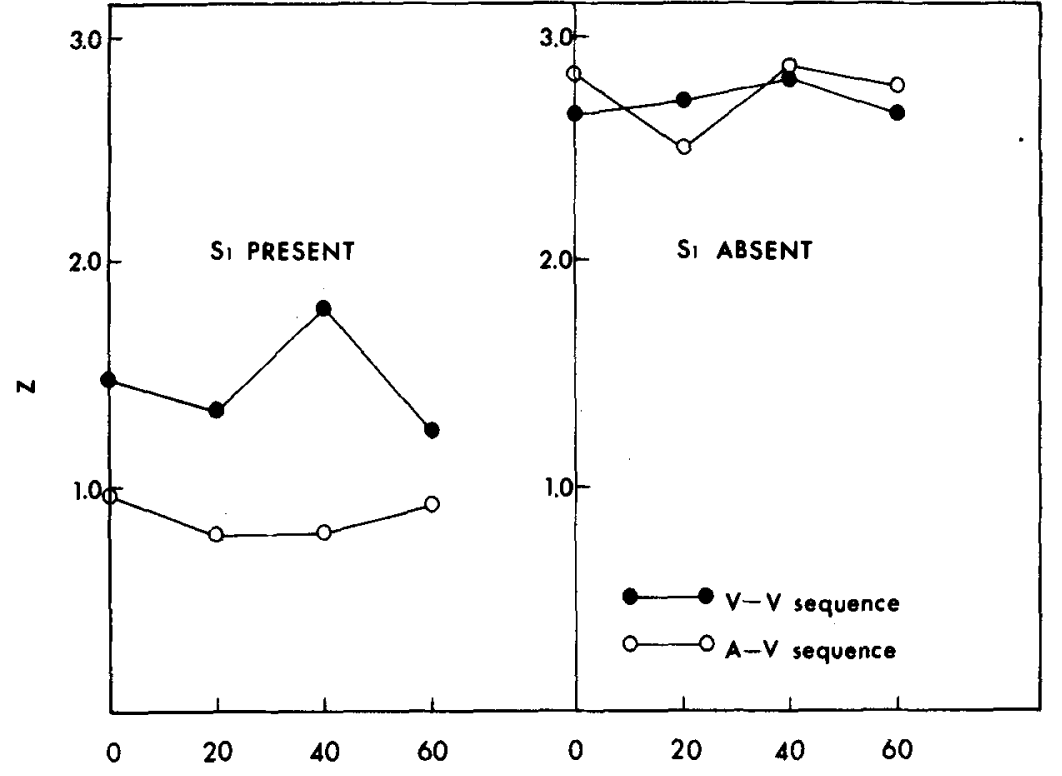

ISI (msec)

Fig. 3. $Z_{2}$ as a function of sequence and ISI, separately for $S_{1}+$ and $S_{1}$-, Experiment 2.

vs $S_{1}$ - were both significant beyond the .01 level; the Sequence Type by ISI interaction was significant beyond the .05 level.

In summary, Experiment 2 suggests that an apparent single-channel effect, as observed in Experiment 1 is obtained only at the expense of greater error rate (lower probability of inhibition). It would thus seem to arise from mechanisms that differ

S. PRESENT

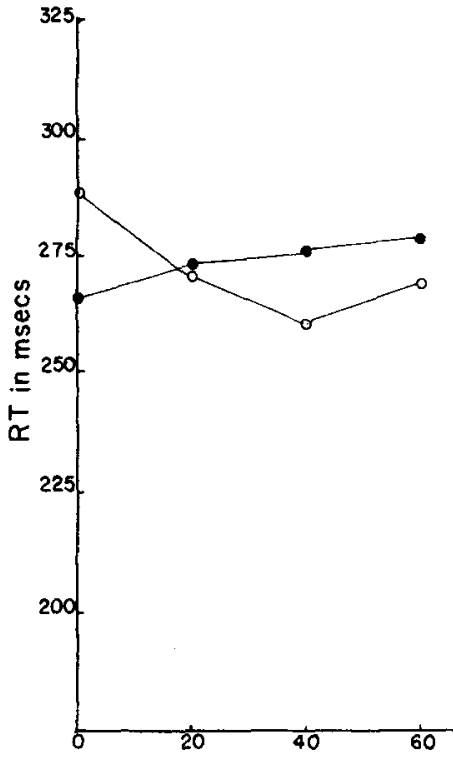

It is thus clear that antagonistic effects are present when intersensory sequences are contrasted with intrasensory sequences. Perhaps the more obvious effect was seen

SI ABSENT

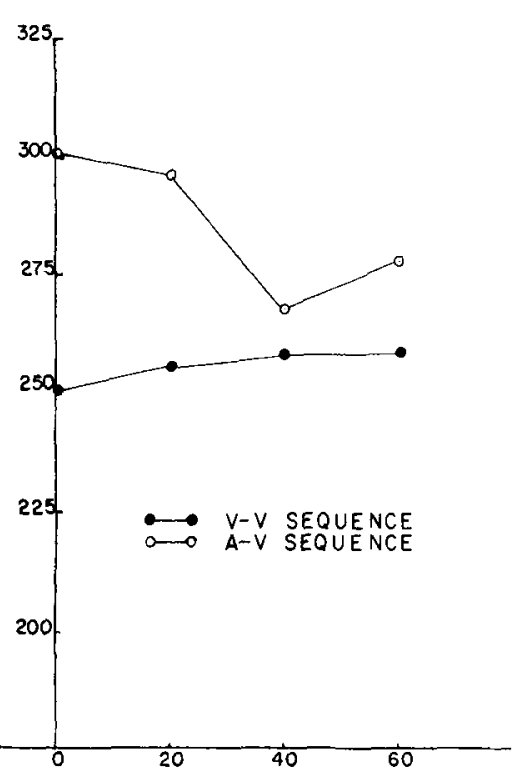

$|S|$ in msecs

Fig. 4. $R T_{2}$ as a function of sequence and ISI, separately for $S_{1}+$ and $S_{1}-$, corrected for $Z_{2}$, Experiment 2. in Experiment 1 and in the uncorrected $\mathrm{S}_{1}+$ data of Experiment 2; RT to a visual event was more rapid when preceded by an auditory as opposed to a visual event. This effect does not seem to be due to the fact that $S_{1}+$ was auditory per se as opposed to visual. Rather, it seems to be due to the fact that the auditory event was more prepotent than the visual as inferred from their respective RTs.

An effect similar to the sequence effect can be seen in the case of $\mathrm{RT}_{2}$ to $S_{1}+\mathrm{vs}$ $S_{1}-$, where $S_{1}-$ would be the limiting case of minimal prepotency. Z and RT jointly varied with both sequence and presence vs absence of $S_{1}$, and RT differences disappeared under error rate correction. In that sense, the RT effects of this type are like the changes in RT as a function of $P(S)$ and pretrial hypotheses reported by Bernstein et al (1970). Some distinction needs to be made, however, to denote that the present pair of RT effects emerged because one specific event (e.g., $S_{1}+$ ) occurred rather than another (e.g., $S_{1}-$ ) and Ss did not know which one would occur in advance. The present events were thus independent of Ss' pretrial expectations. Those reported by Bernstein et al (1970) reflect biases established because $S$ s adjusted to contextual differences present across conditions. Perhaps the terms "triggering," denoting involuntary aspects of performance like the present, and "speed-accuracy trade-off," denoting volutional aspects of Ss' strategy would be heuristic in providing the distinction. A concept like "triggering" seems descriptive of man's limited ability to disjoin consecutive s-r pairings by inhibiting to one and reacting overtly to the other. One means by which this limitation is expressed is that an overt response is less likely to be inhibited when it should not occur but also occurs more rapidly when it should occur as a function of the prepotency of the second event. Clearly, the sequence effect favoring $\mathrm{RT}_{2}$ to $A-V$ does not seem to reflect a true single-channel type of process.

Switching effects were seen in two places of Experiment 2. First there was the Sequence Type by ISI interaction for $\mathrm{RT}_{2}$; $\mathrm{RT}_{2}$ declined across ISI for A.V but not for $V \cdot V$ sequences. The interaction would arise because switching occurs after the clock measuring $\mathrm{RT}_{2}$ starts at ISI $=0 \mathrm{msec}$. As ISI is lengthened, more of the switching time falls prior to the onset of the clock. Note that in Figs. 2 and 4 the decline in $\mathrm{RT}_{2}$ for $\mathrm{A}-\mathrm{V}$ sequences held only up to 40-msec ISI, which is in accord with the strong version of switching theory (Kristofferson, 1967).

The delay in $\mathrm{RT}_{2}$ following $\mathrm{S}_{1}-$ for $\mathrm{A} \cdot \mathrm{V}$ as opposed to $\mathrm{V} \cdot \mathrm{V}$ provides the second 
example of channel-switching effects. This delay was approximately $50 \mathrm{msec}$ at 0 -msec ISI and is also in accord with the strong form of switching theory. 1 The necessary assumptions to make are that an $S_{1}-$ and $S_{2}+$, A.V trial at $0 \mathrm{msec}$ is like a trial on which either $A$ or $V$ can occur, $S$ is aligned to the auditory modality but $\mathrm{V}$ occurs instead. This is the paradigm used by Kristofferson (1967), who discussed why one additional $50-\mathrm{msec}$ quantum is necessary for $S$ to begin the processing of $\mathrm{V}$ above that needed for the parallel type of V.V trial.

Since $\mathrm{RT}_{2}$ did not decline across ISI for $\mathrm{V} \cdot \mathrm{V}$ sequences in Experiment 2, one might question the existence of a refractory effect. A refractory effect would in fact seem to be present since $\mathrm{RT}_{1}$ declined with ISI and $\mathrm{RT}_{2}$ was uniformly longer than
$\mathrm{RT}_{1}$. Sanders (1964) has used a measure of performance based upon $\mathrm{RT}_{1}+\mathrm{RT}_{2}$ to denote Ss' capability of distributing his increased processing ability gained with temporal separation of $S_{1}$ and $S_{2}$ to improve $\mathrm{RT}_{1}$ alone, $\mathrm{RT}_{2}$ alone, or a combination of both. This sum did, in fact, decline with ISI.

\section{REFERENCES}

BERNSTEIN, I. H., BLAKE, R. R., \& CLARK, M. $H$. Sensitivity and decisional effects in the psychological refractory period. Perception \& Psychophysics, 1970, 7, 33-37.

BERNSTEIN, I. H., BLAKE, R. R., \& HUGHES, M. H. Effects of time and event uncertainty upon the psychological refractory period. Perception \& Psychophysics, 1968, 3, 177-184.

KRISTOFFERSON, A. B. Attention and psychological time. Acta Psychologica, 1967, 27, 93-100.

SANDERS, A. F. Selective strategies in the assimilation of successively presented signals. Quarterly Journal of Experimental Psychology, 1964, 16, 368-372.

WELFORD, A. T. The psychological refractory period and the timing of high speed performance: A review and a theory. British Journal of Experimental Psychology, 1952, 43, 2-19.

\section{NOTE}

1. The $25-\mathrm{msec}$ penalty at $0-\mathrm{msec}$ ISI discussed in the introduction is based upon the following premises. $S_{1}$ and $S_{2}$ can be treated as a group which is input simultaneously within a quantum for V-V but $S_{2}$ must await the next quantum for $V$ to be input in A-V. The mean penalty upon $S_{2}$ in $A-V$ is thus the mean unexpired portion of a quantum. As the distribution of $S_{1}$ within a quantum is uniform, the mean unexpired portion is $25 \mathrm{msec}$. These premises seem to be more applicable to event-certain tasks as used in Experiment 1.

(Accepted for publication May 28, 1970.) 